\title{
Analysis of DSRC Service Over-Reach inside an Arched Tunnel
}

\author{
Gilbert Siy Ching, Member, IEEE, Mir Ghoraishi, Member, IEEE, \\ Navarat Lertsirisopon, Jun-ichi Takada, Member, IEEE, Itoji Sameda, \\ Hironori Sakamoto and Tetsuro Imai, Member, IEEE
}

\begin{abstract}
In this paper, the prediction of received power in the out-of-zone of a Dedicated Short Range Communications (DSRC) system operating inside a typical arched highway tunnel is discussed. By conducting wideband directional channel sounding inside the tunnel, the gain, angle-of-arrival and delay of each propagation path are estimated by means of a multidimensional maximum likelihood estimation algorithm from the measured data. Using these estimated parameters and by employing simulations of application antennas according to the DSRC standard, the received power in the out-of-zone is predicted for 2 roadside unit (RSU) antenna positions. The dominant scatterers causing the over-reach of radiated power to the out-ofzone were identified and attributed to the ground and sidewalk. These scatterers can affect the received power level in the outof-zone by as much as $10 \mathrm{~dB}$. It can therefore be concluded that suppressing ground and sidewalk scatterings in the vicinity of RSU by installing composite pavement materials are needed to increase the electromagnetic absorption in order to guarantee DSRC services.
\end{abstract}

Index Terms-DSRC, tunnel propagation, wideband channel measurements, directional measurements, vehicle-roadside communications

\section{INTRODUCTION}

D EDICATED Short Range Communications (DSRC) is a short to medium range communication service intended to improve public safety on the road and also to exchange high speed duplex data between roadside units (RSU) and onboard units (OBU) installed inside vehicles [1]. DSRC applications for intervehicular communications include highway cooperative collision avoidance [2] whereas vehicle-roadside applications include automatic equipment identification and electronic toll collection (ETC) [3], among others. Simultaneous to the development of more components and complex applications for DSRC, the reliability of the system has to be assessed as well [4]-[7]. Specifically, to analyze the DSRC service zone, the system's radio channel has to be characterized.

Among applications of DSRC is the so called location awareness service which can be used in collision avoidance systems as well as security applications [8]. To deploy such

Manuscript received February 1, 2007; revised June 29, 2007.

G. S. Ching, M. Ghoraishi, N. Lertsirisopon, and J. Takada are with the Department of International Development Engineering, Tokyo Institute of Technology, Tokyo 152-8550 Japan (e-mail: gilbert@ap.ide.titech.ac.jp).

I. Sameda was with the Japan Highway Public Corporation and is currently with the West Nippon Expressway Co. Ltd., Japan.

H. Sakamoto is with the Highway Telecom Engineering Co. Ltd., Japan.

T. Imai is with NTT DoCoMo Inc., Japan.

Digital Object Identifier 10.1109/JSAC.2007.071003. services, it is required to limit the radiated power in the service zone to prevent undesired communication by over-reach of the radiated power to the out-of-service zone or simply called out-of-zone. Inside transportation tunnels, the implementation of such an application becomes an even more demanding task. This is because the propagation of radio waves inside tunnels are confined in the tunnel space which can increase the received power in the out-of-zone. To inspect this effect, a detailed knowledge of the communication radio channel inside tunnel is necessary. Although a wide range of researches addressing the radio channel inside tunnel, both theoretically and experimentally, are available, the scenarios in which these analyses have been conducted do not match the DSRC system.

Researches on narrowband radio propagation inside a tunnel focus on signal attenuation and fading characteristics [9]-[15]. Ray-based models were also used to predict the field pattern inside tunnels for both polarizations [16], [17]. More recently, with the assistance of wideband measurements, the root-meansquare (rms) delay spread of the channel could be investigated as well [18]-[21]. In the recent years the directional analysis of the radio propagation channel has attracted lots of attention as the performance of communication systems employing multielement antenna arrays are highly dependent on the spatio-temporal characteristics of the channel. However, in none of the relevant existing researches the spatial attributes of the radio propagation channel inside a tunnel have been investigated. To fill this gap, the authors have conducted a directional wideband measurement campaign utilizing a cylindrical antenna array with dual polarized elements inside an arched tunnel to explore both polarized received paths in angular-delay domains. It has to be noted that the arched tunnel is the typical highway tunnel structure because present construction technology employs the tunnel-boring machine [22] to dig the tunnel and then lower half is leveled, forming an arched cross section.

The analysis of the channel for large coverage communication systems was provided in [23],[24], whereas for the inspection of the over-reach in the DSRC out-of-zone, it is essential to analyze the propagation channel for the nearby area in this particular scenario. For this purpose, two specific scenarios with transmitter (Tx) locations according to the RSU position in Japanese DSRC standard were investigated. The spatio-temporal analysis of the channel accomplished for 204 measurement points covered all transportation lanes in the DSRC out-of-zone. The directional inspection of the 
TABLE I

DSRC TECHNICAL SPECIFICATIONS

\begin{tabular}{ll} 
Parameter & Limits \\
\hline \hline OBU Rx antenna gain & $<10 \mathrm{dBi}$ \\
OBU Rx received power & Inside DSRC zone: -56 to $-40 \mathrm{dBm}$ \\
& Outside DSRC zone: $<-70.5 \mathrm{dBm}$ \\
& $<24.8 \mathrm{dBm}(300) \mathrm{mW}$ \\
RSU Tx transmit power & $<20 \mathrm{dBi}$ \\
RSU Tx antenna gain & right hand circular polarization \\
RSU and OBU polarization & \\
\hline
\end{tabular}

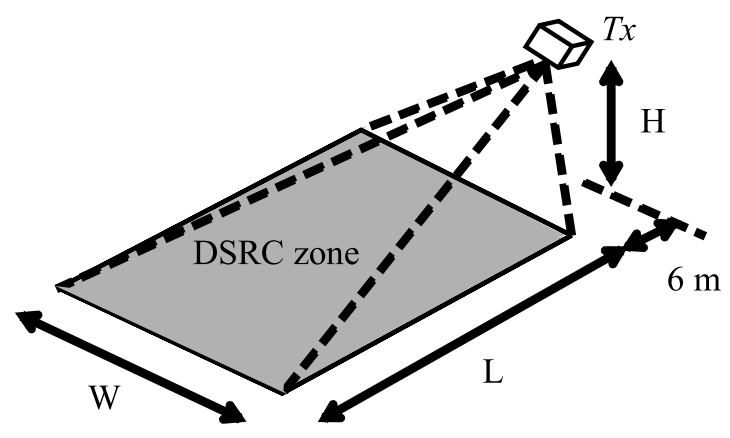

Fig. 1. Illustration of DSRC service zone.

channel allows us to categorize the propagation paths and their scatterers according to the propagation mechanisms. By utilizing the estimated angle-of-arrival (AoA) and angle-ofdeparture (AoD) for the line-of-sight $(\mathrm{LoS})$ and single-bounce paths, the RSU and OBU antenna gains can be included in the prediction of the received power for the out-of-zone. It is shown that, as the LoS and single-bounce path constitute $97 \%$ of the received signal power, this analysis can provide a true over-reach estimate.

Before proceeding to the next sections it is necessary to notify the reader that throughout this paper the term "scattering" is used to express any kind of interaction between propagating wave and the structure of the tunnel or existing objects in the channel unless otherwise mentioned. The paper is organized as follows. The DSRC standard in Japan is discussed in section II while the details of the measurement campaign are presented in section III. In section IV, the parameter estimation process is described. This includes the identification of single-bounce or multiscattering paths and classification of the dominant scatterers. The prediction of the received power inside the tunnel is presented in $\mathrm{V}$ including its analysis, and the conclusions are given in section VI.

\section{DSRC STANDARD IN JAPAN}

The DSRC system standard in Japan is covered in the Association of Radio Industries and Businesses' (ARIB) STDT75 [25] and STD-T88 [26]. It specifies the radio communication interface between a RSU and an OBU installed on a land vehicle. By using time division multiple access-frequency division duplex (TDMA-FDD), fast and large amount of information up to $1024 \mathrm{kbit} / \mathrm{s}$ in the $5.8 \mathrm{Ghz}$ band can be sent to moving vehicles at typical highway speeds. Since 2001, the Japanese DSRC system has been put into use in the form of the ETC system. Other intended services include road and
TABLE II

Measurement Parameters

\begin{tabular}{ll} 
Description & Value \\
\hline \hline Center frequency & $5.2 \mathrm{GHz}$ \\
Bandwidth & $100 \mathrm{MHz}$ \\
Delay resolution & $10 \mathrm{~ns}$ \\
Maximum delay & $6.4 \mu \mathrm{s}$ \\
Tx signal & multitone \\
Tx power & $40 \mathrm{dBm}$ \\
Tx antenna & vertically aligned sleeve dipole \\
Tx antenna height & $8 \mathrm{~m}(\mathrm{Tx} 1), 6.2 \mathrm{~m}(\mathrm{Tx} 2)$ \\
Rx antenna array & cylindrical, 4 rings $\times 24$ dual \\
& polarized patch elements \\
Rx antenna height & $2.5 \mathrm{~m}$ \\
Rx points & 204 points for both Tx1 and Tx2 \\
Synchronization & Cesium clocks \\
\hline
\end{tabular}

traffic information as well as location awareness systems. For these purposes, the service area is limited and within $30 \mathrm{~m}$ from the RSU (assumed Tx here), the received power at the OBU (assumed Rx here) should be from -56 to $-40 \mathrm{dBm}$. Outside the service zone, the received power should be less than $-70.5 \mathrm{dBm}$ to avoid undesired communications. Some technical specifications are shown in Table I and an example of a DSRC service zone is shown in Fig. 1.

\section{Measurement Equipment and Scenario}

Aiming at the over-reach analysis of the DSRC system, a measurement campaign was managed in a highway tunnel in the second Tomei highway, Shizuoka prefecture, Japan. In the following subsections we will describe the employed equipment and the measurement scenario.

\section{A. Equipment}

The RUSK-DoCoMo channel sounder [27] was employed to accomplish the measurements and the related parameters can be found in Table II. The sounding signal is a wideband multitone of $100 \mathrm{MHz}$ bandwidth centered at $5.2 \mathrm{GHz}$, providing a delay resolution of $10 \mathrm{~ns}$. The center frequency of $5.2 \mathrm{GHz}$ was used instead of $5.8 \mathrm{GHz}$ due to the limitation of the channel sounder. A vertical sleeve dipole was employed at the Tx whereas the Rx antenna array was composed of 96 dual-polarized patches constituting 4 rings of 24 dualpolarized elements each. The channel impulse response was taken from each element using a fast RF switch and stored in a hard disk for off-line processing. Cesium clocks were used at both Tx and Rx for synchronization. With the employed $\mathrm{Rx}$ antenna array, both vertically and horizontally polarized received waves could be analyzed. As the Tx antenna is always vertically aligned, vertical and horizontal polarizations are called copolarization and cross-polarization throughout the paper.

\section{B. Scenario}

The tunnel where the measurements were accomplished can accommodate up to 3 car lanes and has a semicircular arched 

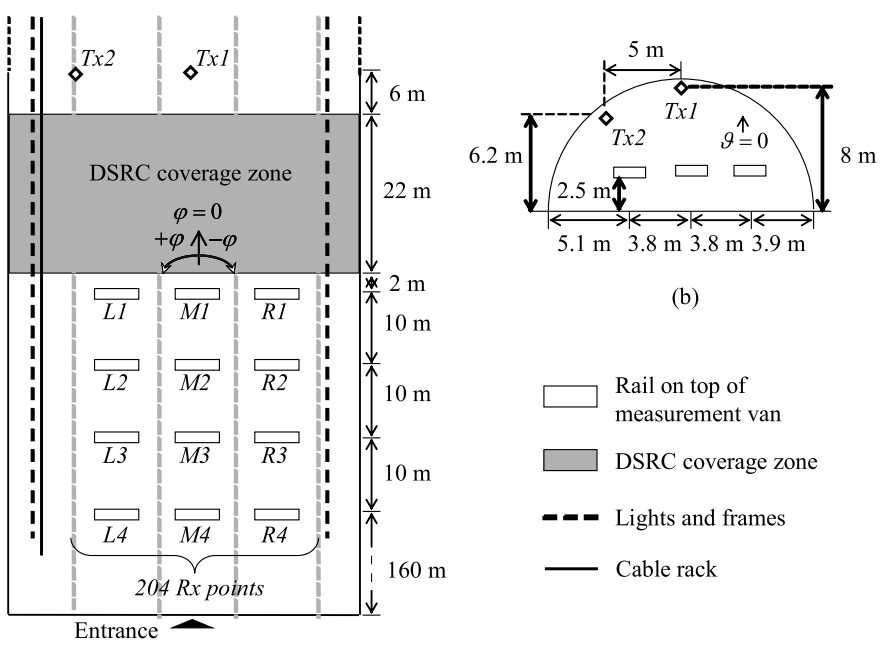

(b)

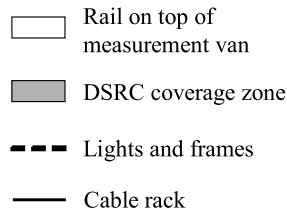

(a)

Fig. 2. (a) Measurement scenario. (b) Front view from tunnel entrance.

cross section. It is $16.6 \mathrm{~m}$ wide on the ground level and the maximum height at the center of the cross section is $8.5 \mathrm{~m}$. At the measurement time, the tunnel was under construction but some structures were already installed as shown in Fig. 2(a). For the scatterer identification in next sections, we classify the tunnel structure as follows:

- ground; the floor of the tunnel that includes the 3 car lanes but excludes the sidewalks.

- left and right sidewalks; located at the sides of the tunnel.

- left and right light-frames; the framing for the lights and cable racks.

- left and right walls; defined as the portion between lightframes and the ground.

- ceiling; defined as the portion above the light-frames.

The measurement was performed in 2 rounds as depicted in Fig. 2(a) to cover the out-of-zone of the DSRC service coverage zone, since we want to identify the propagation mechanism that may cause interference in the out-of-zone. For the first round, the Tx antenna was mounted at the center of the cross section near the ceiling at a height of $8 \mathrm{~m}$ from the ground (Tx1). For the second round, Tx was mounted $5 \mathrm{~m}$ to the left from the center of the tunnel with a height of $6.2 \mathrm{~m}$ from the ground (Tx2). For all rounds, transmitted power was $40 \mathrm{dBm}$ with the Tx antenna aligned vertically and located $220 \mathrm{~m}$ from the entrance of the tunnel.

For the Rx antenna array, it was mounted on a rail on top of the measurement van for a height of $2.5 \mathrm{~m}$ from the ground with line-of-sight (LoS) to the Tx antenna. The measurement van moved to 12 different locations to cover the out-of-zone. The van locations are denoted as L1 to L4, M1 to M4 and $\mathrm{R} 1$ to $\mathrm{R} 4$, corresponding to the left, middle and right lanes respectively. In each van location, the $\mathrm{Rx}$ antenna array moved along the rail in 17 different points with a 2 wavelength separation as shown in Fig. 3. This brings the total number of Rx points to 204 for each Tx case.

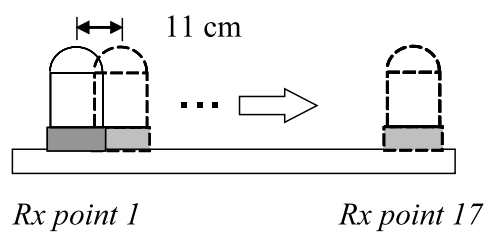

Fig. 3. Illustration of $\mathrm{Rx}$ antenna array position on the rail on top of the measurement van.

\section{Estimation of Propagation PATHS}

\section{A. Parameter Estimation}

A multidimensional maximum likelihood algorithm was employed as the parameter estimator. The signal model for this is composed of discrete paths and diffuse components. For the discrete paths, the channel model equation adopted for static and single-input-multiple-output (SIMO) propagation channel is:

$$
\begin{aligned}
\mathbf{h}(s, \tau, \varphi, \vartheta)=\sum_{l=1}^{L(s)} & {\left[\begin{array}{l}
\gamma_{\mathrm{C}, l} \\
\gamma_{\mathrm{X}, l}
\end{array}\right] } \\
& \times \delta\left(\tau-\tau_{l}\right) \delta\left(\varphi-\varphi_{l}\right) \delta\left(\vartheta-\vartheta_{l}\right)
\end{aligned}
$$

where $s$ refers to the position of the receiver, $L(s)$ is the number of paths in that position, $\gamma_{\mathrm{C}, l}$ and $\gamma_{\mathrm{X}, l}$ are the complex copolarization and cross-polarization path weights respectively, $\tau_{l}$ is the delay, $\varphi_{l}$ is the azimuth and $\vartheta_{l}$ is the coelevation of the angle-of-arrival (AoA) for path $l . \delta(\cdot)$ is the Dirac delta function. The diffuse component is modeled by an exponential function in the delay domain and uniform in the angular domain [28].

In this study, the extracted discrete paths constitute $88 \%$ of the received power. The remaining $12 \%$ of the received power can be considered as diffuse scatterings. Furthermore, having AoA for each extracted path, the Rx antenna array gain could be deconvolved from the estimated data [29].

\section{B. Classification of Extracted Paths}

Assuming the single-bounce, the scattering point for each extracted path could be identified by utilizing the estimated delay and AoA. By employing the AoA, the last scattering object/structure from those introduced in section III-B can be identified whereas the delay helps to confirm if the extracted path is a single-bounce or multiscattering.

Table III shows the extracted paths and their estimated parameters for Tx1, M1-Rx17 where the delay $\tau$ is expressed as the corresponding path length $c \tau$ in meters, where $c$ is the speed of light. The $g_{\mathrm{C}}$ and $g_{\mathrm{X}}$ represent the estimated path gains for the copolarized and cross-polarized components of the extracted path respectively and are defined as

$$
\begin{aligned}
& g_{\mathrm{C}, l}=\left|\gamma_{\mathrm{C}, l}\right|^{2} \\
& g_{\mathrm{X}, l}=\left|\gamma_{\mathrm{X}, l}\right|^{2}
\end{aligned}
$$

The path gain $g_{l}$ is also available by the relation

$$
g_{l}=\left|\gamma_{\mathrm{C}, l}\right|^{2}+\left|\gamma_{\mathrm{X}, l}\right|^{2}
$$


TABLE III

Estimated PATH PARAMETERS FOR TX1,M1-Rx17. 'L' MEANS LeFT WHEREAS 'R' MEANS RIGHT.

\begin{tabular}{|c|c|c|c|c|c|c|c|}
\hline $\begin{array}{l}\text { path } \\
\text { index }\end{array}$ & $\begin{array}{r}g \\
{[\mathrm{~dB}]}\end{array}$ & $\begin{array}{r}c \tau \\
{[\mathrm{m}]}\end{array}$ & $\begin{array}{r}\varphi \\
{[\mathrm{deg}]}\end{array}$ & $\begin{array}{r}\vartheta \\
{[\mathrm{deg}]}\end{array}$ & $\begin{array}{r}g_{\mathrm{C}} \\
{[\mathrm{dB}]}\end{array}$ & $\begin{array}{r}g_{\mathrm{X}} \\
{[\mathrm{dB}]}\end{array}$ & $\begin{array}{c}\text { scatterer } \\
\text { (bounce type) }\end{array}$ \\
\hline 1 & $\begin{array}{r}-80 \\
(-75)\end{array}$ & $\begin{array}{r}31 \\
(31)\end{array}$ & $\begin{array}{r}5 \\
(3)\end{array}$ & $\begin{array}{r}80 \\
(80)\end{array}$ & $\begin{array}{r}-80 \\
(-75)\end{array}$ & -94 & LoS path \\
\hline 2 & -94 & 35 & -26 & 103 & -96 & -98 & $\begin{array}{c}\text { r-sidewalk } \\
\text { (single-bounce) }\end{array}$ \\
\hline 3 & -97 & 34 & 23 & 80 & -100 & -101 & $\begin{array}{l}\text { 1-light-frame } \\
\text { (single-bounce) }\end{array}$ \\
\hline 4 & $\begin{array}{r}-98 \\
(-102)\end{array}$ & $\begin{array}{r}32 \\
(32)\end{array}$ & $\begin{array}{r}5 \\
(3)\end{array}$ & $\begin{array}{r}109 \\
(109)\end{array}$ & $\begin{array}{r}-99 \\
(-102)\end{array}$ & -103 & $\begin{array}{c}\text { ground } \\
\text { (single-bounce) }\end{array}$ \\
\hline 5 & -99 & 33 & -15 & 76 & -100 & -105 & $\begin{array}{l}\text { r-light-frame } \\
\text { (single-bounce) }\end{array}$ \\
\hline
\end{tabular}

The values in parenthesis in Table III represent the theoretical values for LoS and ground specular reflection. The computation of the theoretical LoS path gain assuming a vertically aligned $\lambda / 2$ dipole at the Tx uses the free-space loss factor from the Friis transmission equation given by

$$
g_{f}=(\lambda / 4 \pi r)^{2}
$$

where $r$ is the separation of Tx and Rx. The use of a 5.2 $\mathrm{GHz}$ center frequency instead of $5.8 \mathrm{GHz}$ will result in a $20 \log (5.8 / 5.2)=0.95 \mathrm{~dB}$ increase in the path gains.

If a scattering occurs close to the Tx antenna, it may not be resolvable from LoS as ceiling specular reflections for Tx1. Fig. 4 shows the last scattering point for each of the extracted paths for Tx1, M1-Rx17 in which indexes are consistent with Table III.

\section{Over-Reach Analysis of DSRC Service Zone}

\section{A. Prediction of Received Power Utilizing Directional Param-} eters

The advantage of the directional analysis of the channel is that the effect of the antenna can be deconvolved from propagation paths. Hence the total channel response is expressed as the convolution of the directional channel response and the complex antenna response of the application antennas [29]. Although in this analysis, only the AoAs are estimated, the angles-of-departures (AoDs) $\varphi^{\mathrm{Tx}}$ and $\vartheta^{\mathrm{Tx}}$ can also be indirectly calculated for LoS and single-bounce paths which comprise $62 \%$ and $35 \%$ respectively of the power of the extracted paths for Tx1, and $70 \%$ and $27 \%$ for Tx 2 .

To predict the received power in the out-of-zone, application antennas are assumed at the Tx and Rx sides. In practice, circularly polarized antennas are used in DSRC systems, but in the measurements, we could use only a vertical sleeve dipole in the Tx side due to the limitation of the instruments. Therefore, only the copolarized components are used in the prediction of the mean received power $P_{r}$ at position $s$ as follows:

$$
P_{\mathrm{r}}(s)=\left(\sum_{l=1}^{L^{\prime}(s)} g_{\mathrm{ch}, l}\right) P_{\mathrm{t}}
$$

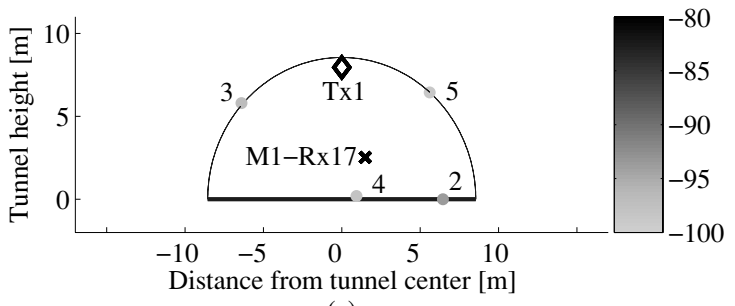

(a)

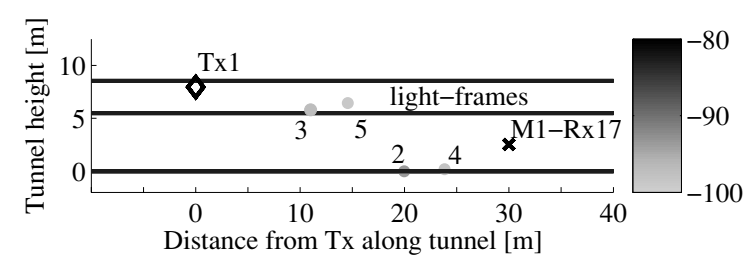

(b)

Fig. 4. The distribution of the scattering points for the case of Tx1, M1Rx17. (a) View from tunnel entrance (b) Tunnel side view. The scale indicates the path gain of the scattering points.

where $L^{\prime}(s)$ is the number of LoS and single-bounce paths in position $s, g_{\mathrm{ch}, l}$ is the radio channel gain of each path given by

$$
g_{\mathrm{ch}, l}=\frac{g_{\mathrm{C}, l}}{g^{\mathrm{D}}\left(\varphi_{l}^{\mathrm{Tx}}, \vartheta_{l}^{\mathrm{Tx}}\right)} g^{\mathrm{Rx}}\left(\varphi_{l}, \vartheta_{l}\right) g^{\mathrm{Tx}}\left(\varphi_{l}^{\mathrm{Tx}}, \vartheta_{l}^{\mathrm{Tx}}\right)
$$

where $g^{\mathrm{Rx}}$ and $g^{\mathrm{Tx}}$ are the gain of the Rx and Tx application antennas respectively, $g^{\mathrm{D}}$ is the gain of the sleeve dipole and $P_{\mathrm{t}}$ is the transmitted power. The cross polarization components were not used since the path gain weighted average of the cross polarization ratio (XPR) for all paths defined as:

$$
\tilde{X}_{\mathrm{C}}=\frac{\sum g_{l} \tilde{X}_{l}}{\sum g_{l}}
$$

where

$$
\tilde{X}_{l}=10 \log \frac{g_{\mathrm{C}, l}}{g_{\mathrm{X}, l}}
$$

is around $14 \mathrm{~dB}$ for both cases. The weighted XPR $\tilde{X}_{\mathrm{C}}$ was used instead of averaging $\tilde{X}_{l}$ since paths with higher path gains are more important [30].

\section{B. Simulation of Rx and Tx Application Antennas}

By working within the limits of the DSRC standard as shown in Table I, a rectangular patch antenna tilted by $\vartheta=45^{\circ}$ along $\varphi=0^{\circ}$ is considered for the Rx application antenna. Its radiation pattern with a maximum gain of $6.4 \mathrm{dBi}$ is shown in Fig. 5. For the Tx application antenna, a uniform received power of $-48 \mathrm{dBm}$ is assumed in the DSRC zone shown in Fig. 2. The maximum gain of the assumed Tx application antenna will be $18 \mathrm{dBi}$ for the Tx 1 case and $19 \mathrm{dBi}$ for the Tx2 case, with a transmitted power of $7 \mathrm{dBm}$. Beyond the DSRC zone, the gain of the assumed Tx application antennas are set to $25 \mathrm{~dB}$ below the maximum gain to limit the received power of LoS in the out-of-zone to less than $-70.5 \mathrm{dBm}$.

Fig. 6 shows the predicted received power for Tx 1 and $\mathrm{Tx} 2$ using Eq. (6). The dots indicate that the received power at that position is above the $-70.5 \mathrm{dBm}$ threshold value set by 


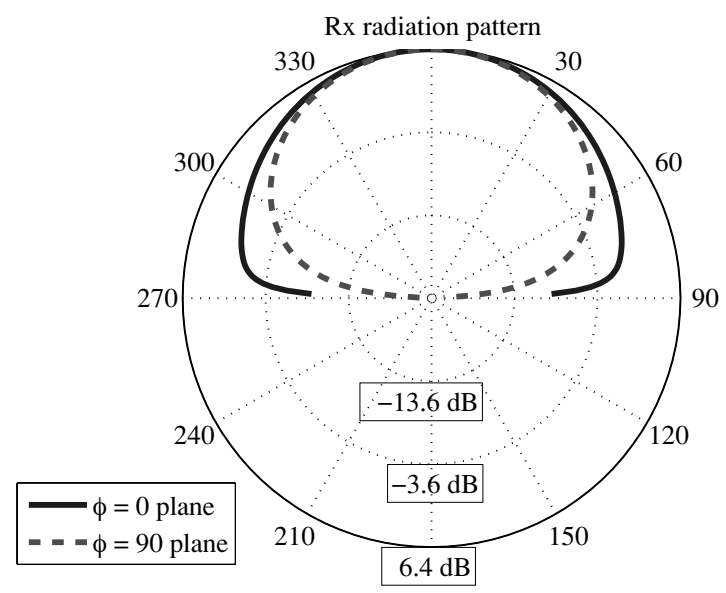

Fig. 5. Radiation pattern of Rx application antenna.

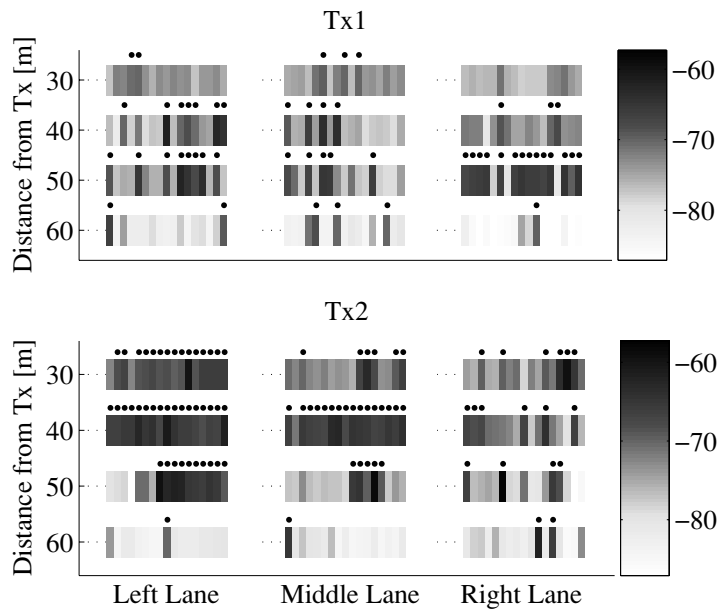

Fig. 6. Predicted received power comparison for $\mathrm{Tx} 1$ and $\mathrm{Tx} 2$. The dots indicate that the received power at that position is above the $-70.5 \mathrm{dBm}$ limit set by the DSRC standard outside the service zone. The scale is the predicted received power in $\mathrm{dBm}$.

the DSRC standard outside the service zone. Although the received power due to the $\operatorname{LoS}$ path in the out-of-zone is less than $-70.5 \mathrm{dBm}$, the contribution of other paths from scatterings inside the tunnel can increase the total received power beyond the limit in that position.

\section{Scatterer Analysis}

To analyze which scatterers have high power contribution to the received power, we define $\tilde{g}_{\mathrm{SC}}$, the scatterer class gain in $\mathrm{dB}$ as follows:

$\tilde{g}_{\mathrm{SC}}=10 \log \left(g_{\mathrm{ch}, \mathrm{LoS}}+\sum_{l \in C_{j}} g_{\mathrm{ch}, l}\right)-10 \log g_{\mathrm{ch}, \mathrm{LoS}}$

where $C_{j}$ corresponds to the $j$-th scatterer class set to which the extracted path $l$ belongs. This equation shows the increase of the received power from LoS due to classified scatterings. Note that fading can occur due to the combinations of the paths. To get the expected mean value of this fluctuation, one can consider only adding the gains of each path [31] as done in Eqs. (6) and (10).

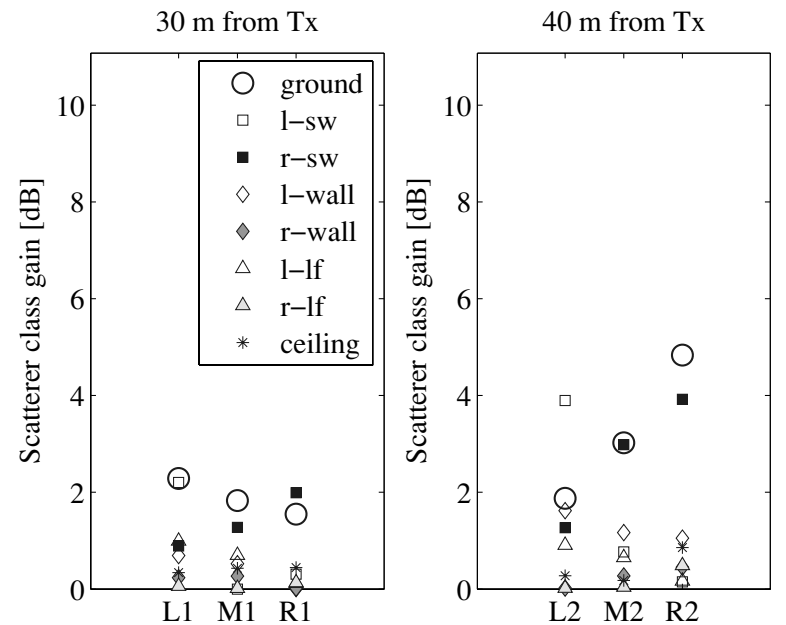

Fig. 7. Scatterer class gain for Rx at 30 meters (left lane (L1), middle lane (M1), right lane (R1)) and 40 meters (left lane (L2), middle lane (M2), right lane (R2)) away from Tx1.
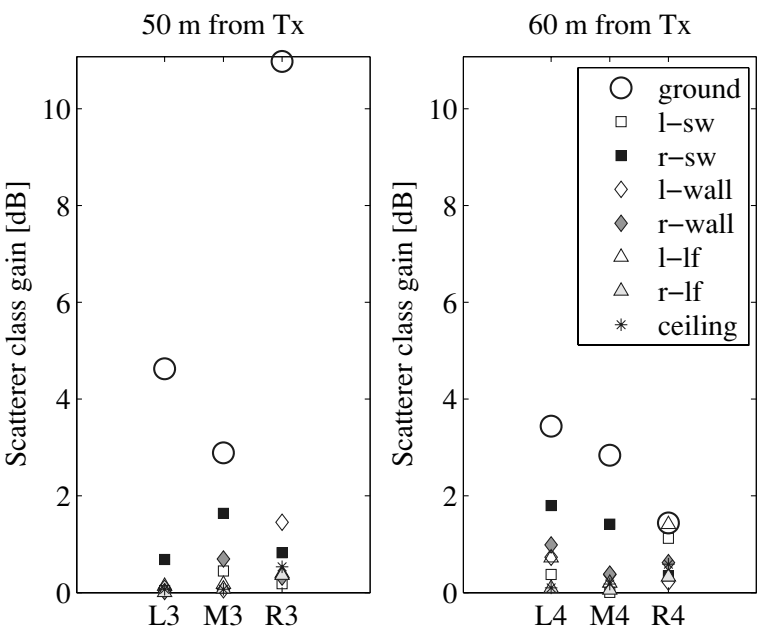

Fig. 8. Scatterer class gain for Rx at 50 meters (left lane (L3), middle lane (M3), right lane (R3)) and 60 meters (left lane (L4), middle lane (M4), right lane (R4)) away from Tx1.

Figs. 7 and 8 show the scatterer class gain for Tx 1 averaged at each of the 12 measurement van locations. From these figures, we observe the following:

- ground, left and right sidewalk scatterers are generally dominant in any position.

- at $30 \mathrm{~m}$ and $40 \mathrm{~m}$ from the Tx, the left sidewalk scatterers are also dominant in the left lane (L1 and L2) whereas right sidewalk scatterers are dominant in the right lane (R1 and R2).

- as the Tx to Rx separation increases, the LoS gain decreases and the scatterer class gain can become higher than the LoS gain, especially for the scatterers inside the service zone.

- at $60 \mathrm{~m}$ from Tx, both the LoS and scatterer class gains are low and do not affect the received power much as shown in Fig. 6.

For Tx2, Figs. 9 and 10 show the scatterer class gain averaged at each of the 12 measurement van locations. In 


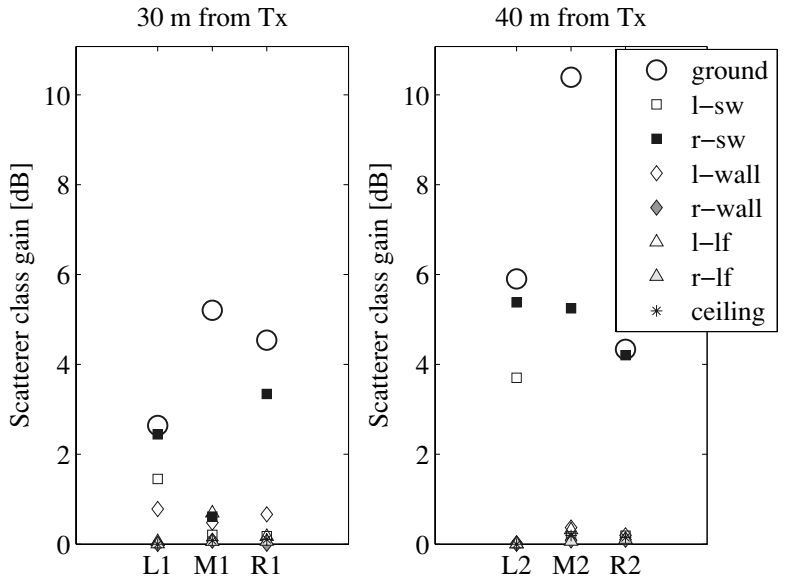

Fig. 9. Scatterer class gain for Rx at 30 meters (left lane (L1), middle lane (M1), right lane (R1)) and 40 meters (left lane (L2), middle lane (M2), right lane (R2)) away from $\mathrm{Tx} 2$.
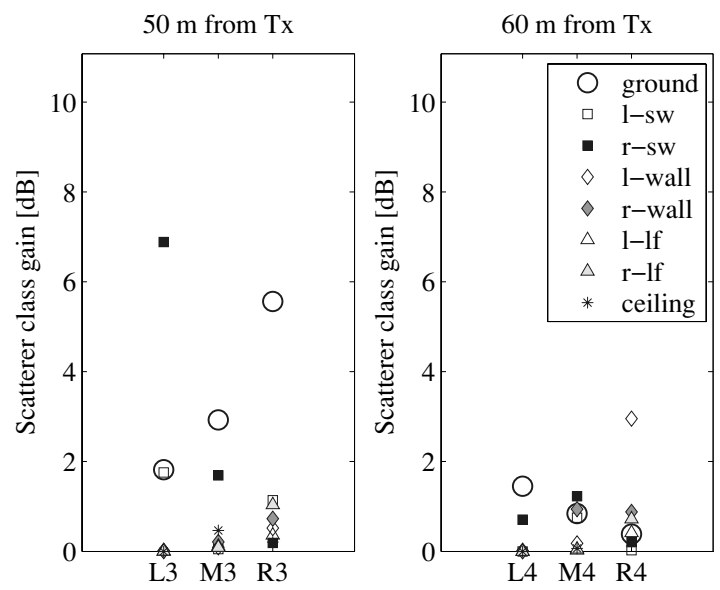

Fig. 10. Scatterer class gain for Rx at 50 meters (left lane (L3), middle lane (M3), right lane (R3)) and 60 meters (left lane (L4), middle lane (M4), right lane (R4)) away from $\mathrm{Tx} 2$.

addition to the observations made for Tx 1 , we observe that for $\mathrm{Tx} 2$, even in the left lanes, the right sidewalk scatterers have larger contribution than the left sidewalk scatterers. This might be due to the position of Tx2 which makes some scattering from the left sidewalk close to the LoS path and difficult to be distinct.

Figs. 11 to 14 present top views of ground and sidewalk scatterer positions for both Tx cases. We note the following:

- as Tx to Rx separation increases, the ground and sidewalk scatterings occur in a larger distance from Tx. This can be interpreted as specular-like scatterings.

- for Tx1 case, scatterers are distributed more uniformly across the tunnel, whereas for $\mathrm{T} \times 2$ case, the number of sidewalk scatterings at the right and ground scatterings at the left are higher.

\section{Suppression of Scatterers}

The dominant influence of the scattering received power in the out-of-zone hints the possibility of employing absorbers to acquire a better performance for the DSRC system. Other

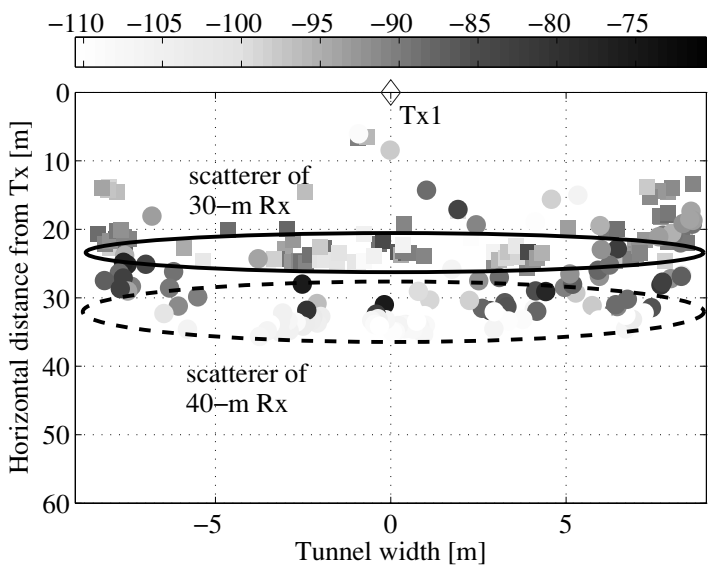

Fig. 11. Tunnel top view of ground and sidewalk scatterers for Tx1. For Rx points 30 and $40 \mathrm{~m}$ away from the Tx, its scatterers are represented by squares and circles respectively. The scale is the radio channel gain in $\mathrm{dB}$.

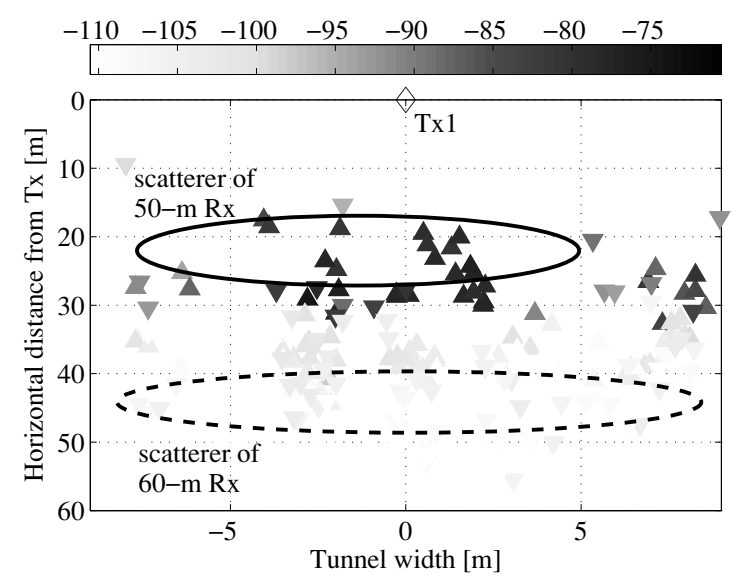

Fig. 12. Tunnel top view of ground and sidewalk scatterers for Tx1. For Rx points 50 and $60 \mathrm{~m}$ away from the Tx, its scatterers are represented by triangles and inverted triangles respectively. The scale is the radio channel gain in $\mathrm{dB}$.

non-tunnel scenarios which use absorbers to improve system performance include [32] and [33]. We evaluated the influence of excluding dominant scatterer classes on the total received power. Fig. 15 shows the received power excluding the ground scatterings. A significant reduction is observed for the Tx 1 case whereas for some points in the Tx 2 case, the received power is still above the DSRC limit. In Fig. 16 scatterings from sidewalks are excluded as well. It can be observed that the received power in most positions is under the required threshold.

\section{CONCLUSION}

Location awareness service of DSRC systems requires that radiated power be within a certain service zone. Even though the LoS path to the out-of-zone is below the threshold level, scatterings can increase the received power. In this paper, the prediction of received power in the out-of-zone of a DSRC system operating inside a typical highway arched tunnel was analyzed. A wideband directional channel sounding was con- 


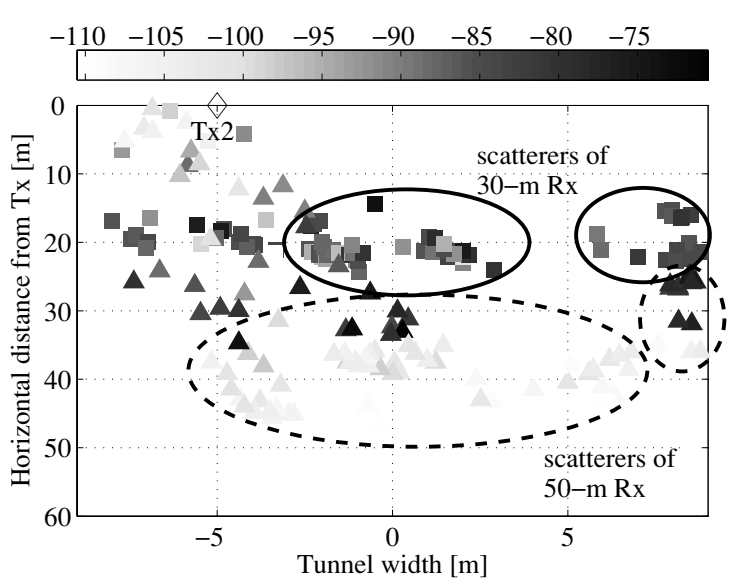

Fig. 13. Tunnel top view of ground and sidewalk scatterers for Tx2. For Rx points 30 and $50 \mathrm{~m}$ away from the Tx, its scatterers are represented by squares and triangles respectively. The scale is the radio channel gain in $\mathrm{dB}$.

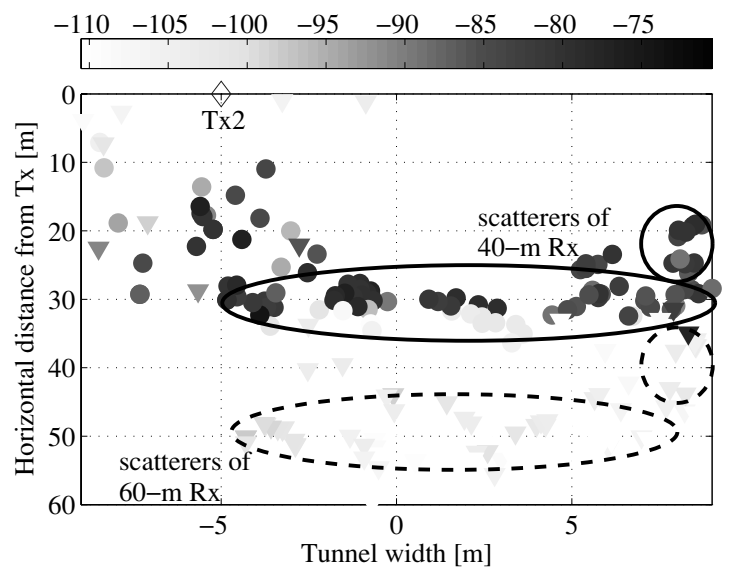

Fig. 14. Tunnel top view of ground and sidewalk scatterers for Tx2. For Rx points 40 and $60 \mathrm{~m}$ away from the Tx, its scatterers are represented by circles and inverted triangles respectively. The scale is the radio channel gain in $\mathrm{dB}$.

ducted inside the tunnel to assess the coverage in the out-ofzone. Thus limitations of simulation models such as influence of scattering from smaller objects could be overcome. The parameters of the radio propagation paths were then estimated by a multidimensional-maximum-likelihood algorithm. This leads to the separation of the $\operatorname{LoS}$ and single-bounce paths from the effects of the measurement antennas. Application antennas that satisfy the threshold values of the DSRC standard are used together with the estimated parameters to predict the received power in the out-of-zone for two Tx antenna positions. The dominant scatterings that cause the over-reach of radiated power to the out-of-zone were then identified and attributed to the ground and sidewalk scatterers. These scatterings can increase the received power level by as much as $10 \mathrm{~dB}$. From these results, it is concluded that the use of composite pavement material to increase the electromagnetic absorption is essential for DSRC services.

It is noted that the signal symbol period in the Japanese DSRC standard is set around $1 \mu \mathrm{s}$. Moreover the delay spread from the measurements inside the arched tunnel is around 20

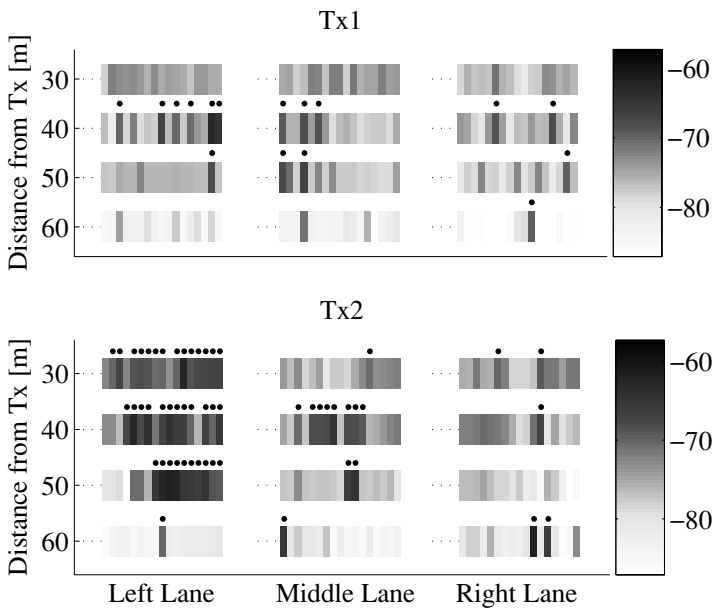

Fig. 15. Predicted received power comparison without ground scatterer contribution for Tx1 and Tx2. The dots indicate that the received power at that position is above the $-70.5 \mathrm{dBm}$ limit set by the DSRC standard outside the service zone. The scale is the predicted received power in $\mathrm{dBm}$.
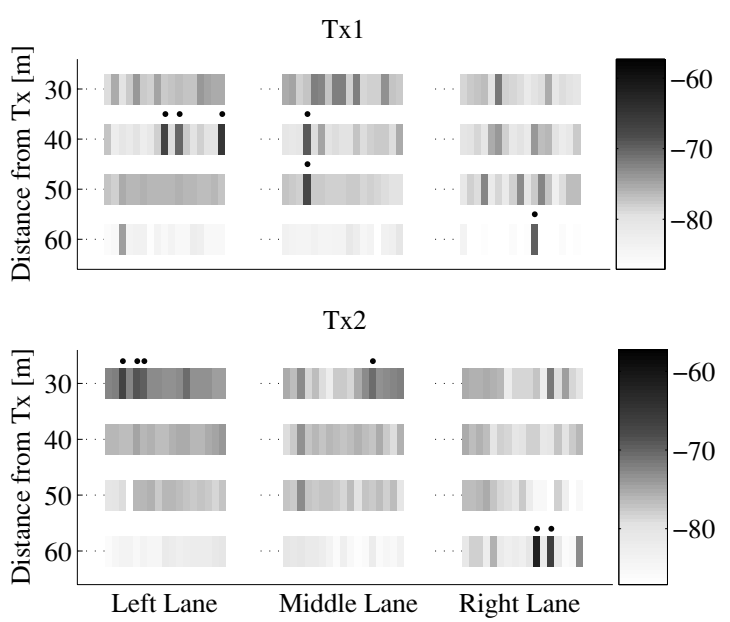

Fig. 16. Predicted received power comparison without ground and sidewalk scatterer contributions for Tx1 and Tx2. The dots indicate that the received power at that position is above the $-70.5 \mathrm{dBm}$ limit set by the DSRC standard outside the service zone. The scale is the predicted received power in $\mathrm{dBm}$.

ns [34]. This is well below one tenth of the symbol period which means the channel can be modeled as narrowband. Also the analysis presented in this paper shows that over-reach can occur for the DSRC system up to $50 \mathrm{~m}$. These results are well applicable to systems with a symbol period larger than $200 \mathrm{~ns}$ and service zones of less than $50 \mathrm{~m}$ away. Further discussions for a larger Tx and Rx separations can be found in [24].

For tunnels with other cross-section shapes, the scatterings from ceiling, light-frame and wall will be different but the dominant single-bounce ground and sidewalk scatterings are preserved, hence over-reach analysis discussed in this paper is pertinent. Finally it has to be mentioned that the examined scenario is the DSRC system in a highway tunnel, for which the distance between vehicles should be in the range of 100 $\mathrm{m}$ for the safety reasons. This means having a front vehicle on the same lane in the service zone is less probable, thus contribution from other vehicles on the same lane is minimal. To assess the impact of the vehicles on adjacent lanes, further research should be considered. 


\section{REFERENCES}

[1] Dedicated Short Range Communications. Available: http://grouper.ieee.org/groups/scc32/dsrc/

[2] S. Biswas, R. Tatchikou, and F. Dion, "Vehicle-to-vehicle wireless communication protocols for enhancing highway traffic safety," IEEE Communications Magazine, vol. 44, pp. 74-82, Jan. 2006.

[3] W.-Y. Shieh, W.-H. Lee, S.-L. Tung, B.-S. Jeng, and C.-H. Liu, "Analysis of the optimum configuration of roadside units and onboard units in dedicated short-range communication systems," IEEE Trans. Intelligent Transportation Systems, vol. 7, pp. 565-571, Dec. 2006.

[4] K. Nishikawa, "Land vehicle antennas ," IEICE Trans. Commun., vol. E86-B, no. 3, pp. 993-1004, Mar. 2003.

[5] T. Sawaya, Y. Okamoto, K. Iigusa, M. Taromaru, and T. Ohira, "Consecutive radio-zone DSRC system with an in-vehicle reactance diversity antenna," in Proc. IEEE Intelligent Transportation Systems, pp. 492-497, Sep. 2005.

[6] D. Jiang, V. Taliwal, A. Meier, W. Holfelder, and R. Herrtwich, "Design of $5.9 \mathrm{ghz}$ dsrc-based vehicular safety communication," IEEE Wireless Communications, vol. 13, pp. 36-43, Oct. 2006.

[7] A. Visser, H.H. Yakali,A.-J. van der Wees, M. Oud, G.A. van der Spek, and L.O. Hertzberger, "A hierarchical view on modeling the reliability of a DSRC link for ETC applications," IEEE Trans. Intelligent Transportation Systems, vol. 3, pp. 120-129, June 2002.

[8] J.P. Hubaux, S. Capkun, and J. Luo, "The security and privacy of smart vehicles," IEEE Security \& Privacy Magazine, vol. 2, pp. 49-55, May 2004.

[9] A. G. Emslie, R. L. Lagace, and P. F. Strong, "Theory of the propagation of UHF radio waves in coal mine tunnels," IEEE Trans. Antennas Propag., vol. AP-23, pp. 192-205, Mar. 1975.

[10] Y. Yamaguchi, T. Abe, T. Sekiguchi, and J. Chiba, "Attenuation constants of UHF radio waves in arched tunnels," IEEE Trans. Microw. Theory Tech., vol. MTT-33, no. 8, pp. 714-718, Aug. 1985.

[11] K. Fujimori and H. Arai, "Propagation characteristics in tunnels including base station antenna," Electron. and Commun. in Japan (Part I. Commun.), vol. 84, iss. 4, pp. 1-10, April 2001.

[12] K. Uchida, H. Nose, H. Maeda, and T. Matsunaga, "Theoretical and experimental study of propagation in 3D tunnels," IEICE Trans. Commun., vol. E87-B, no. 10, pp. 3044-3049, Oct. 2004.

[13] D. G. Dudley, and S. F. Mahmoud, "Linear source in a circular tunnel," IEEE Trans. Antennas Propag., vol. 54, no. 7, pp. 2034-2047, July 2006.

[14] T. S. Wang, and C. F. Yang, "Simulations and measurements of wave propagations in curved road tunnels for signals from GSM base stations," IEEE Trans. Antennas Propag., vol. 54, no. 9, pp. 2577-2584, Sep. 2006.

[15] C. Briso-Rodríguez, J. M. Cruz, and J. I. Alonzo, "Measurements and modeling of distributed antenna systems in railway tunnels," IEEE Trans. Veh. Technol., to be published.

[16] D. Didascalou, T. Schäfer, F. Weinmann, and W. Wiesbeck, "Ray-density normalization for ray-optical wave propagation modeling in arbitrarily shaped tunnels," IEEE Trans. Antennas Propag., vol. 48, no. 9, pp. 13161325, Sep. 2000.

[17] T. Imai, "Prediction of propagation characteristics in tunnels using raytracing method," IEICE Trans. Commun. (Japanese Edition), vol. J85-B, no. 2, pp. 216-226, Feb. 2002.

[18] J. Molina-Garcia-Pardo, J. Rodríguez, and L. Juan-Llácer, "Wide-band measurements and characterization at $2.1 \mathrm{GHz}$ while entering in a small tunnel," IEEE Trans. Veh. Technol., vol. 53, no. 6, pp. 1794-1799, Nov. 2004.

[19] Y. P. Zhang and H. J. Hong, "Ray-optical modelling of simulcast radio propagation channels in tunnels," IEEE Trans. Veh. Technol., vol. 53, no. 6, pp. 1800-1808, Nov. 2004.

[20] M. Liénard and P. Degauque, "Natural wave propagation in mine environments," IEEE Trans. Antennas Propag., vol. 48, no. 9, pp. 13261339, Sep. 2000.

[21] C. Nerguizian, C. Despins, S. Affès, and M. Djadel, "Radio-channel characterization of an underground mine at $2.4 \mathrm{GHz}$," IEEE Trans. Wireless Commun., vol. 4, no. 5, pp. 2441-2453, Sep. 2005.

[22] T. Konda, "Shield tunneling method," Civil Engineering, Japan Society of Civil Engineers, vol. 39, pp. 23-27, July 2001.

[23] G. S. Ching, M. Ghoraishi, N. Lertsirisopon, J. Takada, T. Imai, I. Sameda, and H. Sakamoto, "Wideband directional radio propagation channel analysis inside an arched tunnel," in Proc. 17th IEEE Intl. Sym. on Personal, Indoor and Mobile Radio Comm. (PIMRC 2006), Sep. 2006.

[24] G. S. Ching, M. Ghoraishi, M. Landmann, N. Lertsirisopon, J. Takada, T. Imai, I. Sameda, and H. Sakamoto, "Wideband polarimetric directional propagation channel analysis inside an arched tunnel," submitted to IEEE Trans. Antennas Propag.,
[25] "Dedicated Short-Range Communication System," ARIB STD-T75, Ver.1.0, Sep. 2001.

[26] "DSRC Application Sub-Layer," ARIB STD-T88, Ver.1.0, May 2004.

[27] Rusk-Vector Channel Sounder. Available: http://www.channelsounder.de/

[28] R. S. Thomä, M. Landmann, G. Sommerkorn, and A. Richter, "Multidimensional high-resolution channel sounding in mobile radio," in Proc. 21 st IEEE Inst. Meas. Technol. Conf., pp. 257-262, May 2004.

[29] L. Correia, Wireless Flexible Personalised Communications: COST 259: European Co-operation in Mobile Radio Research, John Wiley and Sons, 2001.

[30] M. Landmann, K. Sivasondhivat, J. Takada, I. Ida, and R. Thomä, "Polarization behavior of discrete multipath and diffuse scattering in urban environments at $4.5 \mathrm{GHz}$," EURASIP J. Wireless Commun. and Networking, vol. 2007, Article ID 57980, 16 pages, Jan 2007.

[31] F. Ikegami, S. Yoshida, T. Takeuchi, and M. Umehira, "Propagation factors controlling mean field strength on urban streets," IEEE Trans. Antennas Propag., vol. ap-23, no. 8, pp. 822-829, Aug. 1984.

[32] R. K. Pokharel, M. Toyota, and O. Hashimoto, "Analysis on effectiveness of wave absorbers to improve DSRC electromagnetic environment on express highway," IEEE Trans. Microw. Theory Tech., vol. 53, no. 9, pp. 2726-2731, Sep. 2005.

[33] K. Haneda, J. Takada, T. Iwata, Y. Wakinaka, and T. Kunishima, "Experimental determination of propagation paths for the ETC systemequipment development and field test- ," IEICE Trans. Fundamentals, vol. E87-A, no. 11, pp. 3008-3015, Nov. 2004.

[34] G. S. Ching, M. Ghoraishi, N. Lertsirisopon, J. Takada, I. Sameda, R. Soma, H. Sakamoto, and T. Imai, "Tunnel propagation channel characterization for DSRC applications," submitted to 2007 Asia-Pacific Microwave Conference

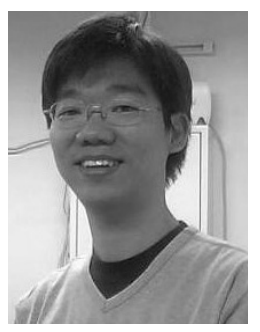

Gilbert Siy Ching (S'98-M'07) was born in Manila, Philippines in 1974. He received his B.S. and M.S. degrees in electrical engineering from the University of the Philippines in 1996 and 2003, respectively and was also an instructor at the same university from 1996 to 2003. Presently, he is pursuing his doctoral degree at the Tokyo Institute of Technology. His current interests are in radio propagation channel measurements, analysis and modeling.

Mr. Ching was the recipient of the best student paper award from the area of Asia Pacific at the 17th International Symposium on Personal, Indoor and Mobile Radio Communications (PIMRC '06). He is a student member of The Institute of Electronics, Information and Communication Engineers (IEICE), Japan.

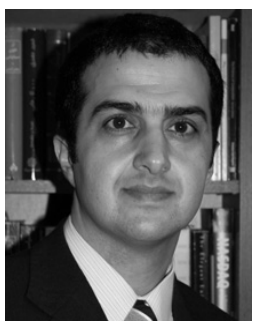

Mir Ghoraishi (S'99-M'05) received B.E. from Isfahan University of Technology, Esfahan, Iran, and M.E. from Amirkabir University of Technology, Tehran, Iran, both in electrical engineering in 1993 and 1999 respectively.

From 1999 to 2004 he was a Ph.D. student in Tokyo Institute of Technology, Department of Electric and Electronic Engineering. Since 2004 he is a researcher with Tokyo Institute of Technology, Department of International Development Engineering. His research interests are wireless localization and object tracking, wireless radio propagation channel measurement and modeling, array antenna design and super-resolution parameter estimation algorithm. He is a member of the Institute of Electronics, Information and Communication Engineers of Japan (IEICE).

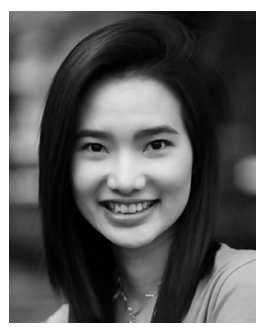

Navarat Lertsirisopon was born in Bangkok, Thailand, in 1981. She received her B.E. degree from Sirindhorn International Institute of Technology, Thammasat University, Bangkok, Thailand in 2002 and received her M.E. degree from Tokyo Institute of Technology, Tokyo, Japan in 2006. Presently, she is studying towards the D.E. degree in Tokyo Institute of Technology, Tokyo, Japan. Her current research interest is propagation prediction modeling in wireless communication systems.

Ms. Lertsirisopon is a student member of The Institute of Electronics, Information and Communication Engineers (IEICE), Japan. 


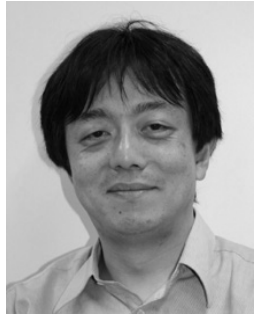

Jun-ichi Takada (S'89-M'93) received the B.E., M.E., and D.E. degrees from the Tokyo Institute of Technology, Tokyo, Japan, in 1987, 1989, and 1992 , respectively. From 1992 to 1994, he was a Research Associate Professor with Chiba University, Chiba, Japan.

From 1994 to 2006, he was an Associate Professor with Tokyo Institute of Technology. Since 2006, he has been a Professor with Tokyo Institute of Technology. His current interests are wireless propagation and channel modeling, array signal processing, UWB radio, cognitive radio, applied radio instrumentation and measurements, and ICT for international development.

Dr. Takada is a member of IEICE,ACES, and the ECTI Association Thailand.

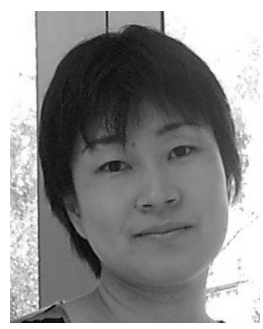

Itoji Sameda was born in Oita, Japan in 1968. She received her B.S and M.S degrees in electrical and electronics engineering from Nagasaki University, Japan in 1992 and 1994, respectively. She joined the Japan Highway Public Corporation in 1994. In 2005 , the company was privatized and she is now with the ETC (Electronic Toll Collection) system section, Expressway Operation department, Kansai branch of West Nippon Expressway Co. Ltd. She is interested in wireless communication systems.
Hironori Sakamoto is with the Highway Telecom Engineering Co. Ltd., Japan.

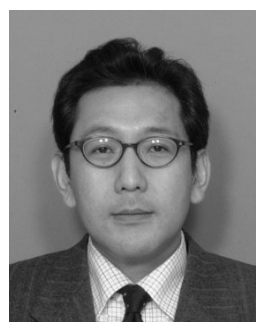

Tetsuro Imai (M'93) was born in Tochigi, Japan, in 1967. He received his B.S. and Ph.D. degrees from Tohoku University, Japan, in 1991 and 2002, respectively.

He joined the Wireless System Laboratories of Nippon Telegraph and Telephone Corporation (NTT), Kanagawa, Japan, in 1991. Since then, he has been engaged in the research and development of radio propagation and system design for mobile communications. He is now Manager of the Radio Access Network Development Department, NTT DoCoMo, Inc., Kanagawa, Japan.

Dr. Imai is a Member of the Institute of Electronics, Information and Communication Engineers of Japan (IEICE). 